\title{
Age trends in direct medical costs of pediatric asthma: a population study
}

\author{
Wenjia Chen ${ }^{1}$, Hamid Tavakoli ${ }^{1}$, J FitzGerald ${ }^{1}$, Padmaja Subbarao ${ }^{2}$, Turvey Stuart $^{1}$, and \\ Mohsen Sadatsafavi ${ }^{1}$ \\ ${ }^{1}$ The University of British Columbia \\ ${ }^{2}$ University of Toronto
}

March 31, 2021

\begin{abstract}
Background: Quantifying age trends in healthcare costs of pediatric asthma leads to better understanding of the natural history of the disease and informed decision-making on the allocation of healthcare resources. Methods: We identified children with incident asthma from the health administrative data of British Columbia, Canada (Jan 1998 to Dec 2015), and followed them from their first diagnosis of asthma or wheezing until age 18. We estimated direct medical costs (in 2016 Canadian dollars $[\$]$ ), including inpatient and outpatient encounters and pharmacy costs, attributed to asthma (primary outcome) and other respiratory diseases (secondary outcome). We assessed the impact of sex and socioeconomic status on age trends, adjusting for calendar effect. Results: The final analysis included 44,552 children with asthma (62\% boys). From age 0 to 18 , costs of asthma/wheezing and other respiratory conditions decreased from $\$ 1,036$ to $\$ 29 /$ child-year, and from $\$ 1,145$ to $\$ 31 /$ child-year, respectively. Children under 3 years of age incurred 4 -fold higher costs for asthma/wheezing and other respiratory conditions. In particular, costs of asthma hospitalizations were 10 times higher in this age group compared to older children. Age trends were generally similar between sex groups and across socioeconomic status. However, medication costs for asthma/wheezing decreased in boys, whereas those in girls declined during childhood but increased during adolescence. Conclusions: The highest costs of pediatric asthma are concentrated in children younger than 3. Age trends were generally consistent between sex and across socioeconomic status.
\end{abstract}

\section{Hosted file}

pediatric.asthmacost_pai_lte_fin.pdf available at https://authorea.com/users/398020/articles/ 516081-age-trends-in-direct-medical-costs-of-pediatric-asthma-a-population-study

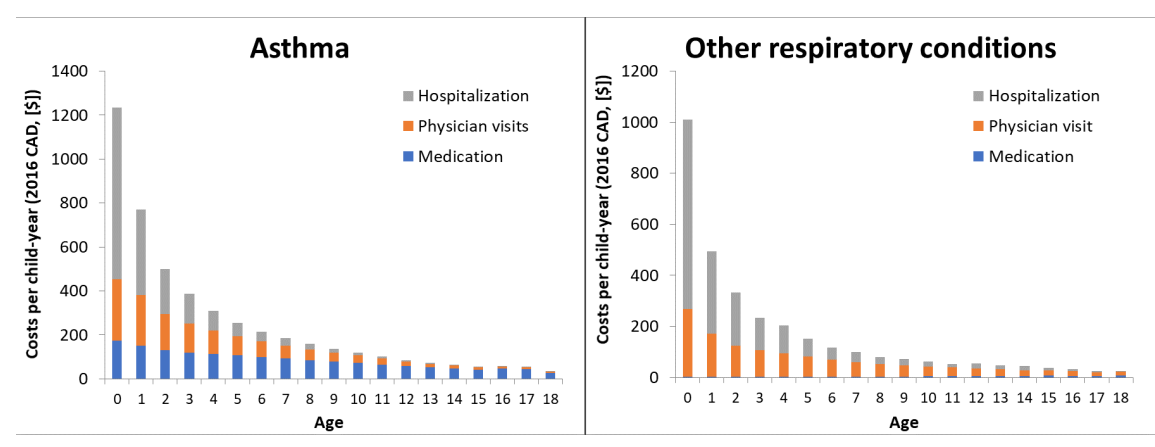

